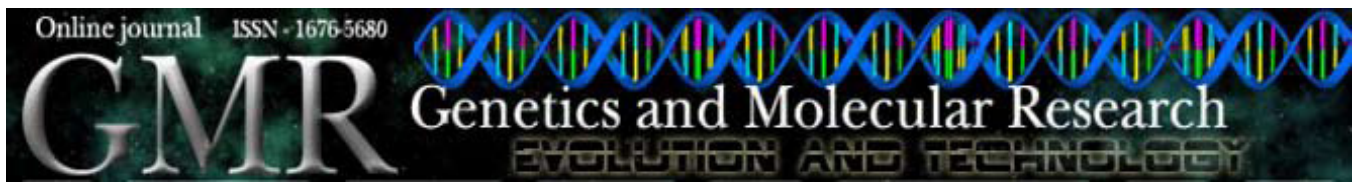

\title{
Identification and characterization of conserved microRNAs and their target genes in wheat (Triticum aestivum)
}

\author{
Z.J. Yin and F.F. Shen \\ State Key Laboratory of Crop Biology, College of Agronomy, \\ Shandong Agricultural University, Taian, Shandong, China \\ Corresponding author: F.F. Shen \\ E-mail: ffshen@sdau.edu.cn
}

Genet. Mol. Res. 9 (2): 1186-1196 (2010)

Received February 25, 2010

Accepted March 29, 2010

Published June 22, 2010

DOI 10.4238/vol9-2gmr805

\begin{abstract}
MicroRNAs (miRNAs) are non-coding small RNAs that regulate gene expression by translational repression or transcript degradation. A large number of miRNAs have been identified from model plant species; however, the character of conserved miRNAs is poorly understood. We studied 42 miRNA families that are conserved within the plant kingdom, using the miRBase database. Some conserved miRNA families were found to be preferentially expressed in dicots relative to monocots, especially miR403, miR472 and miR479. Using an improved homology search-based approach and the conserved miRNAs as the query set, 34 conserved miRNAs and the miR482 family were identified in wheat. Forty-six wheat mRNAs were predicted as their putative target genes. Most conserved wheat miRNAs were found to retain homologous target interactions and have analogous molecular functions. The miR172 displayed a wheat-specific function and was found to have an additional target interaction with succinyl-CoA ligase.
\end{abstract}


We concluded that although miRNAs are conserved, the expression and function of some have drifted during long periods of plant evolution.

Key words: MicroRNAs; Wheat; Target genes; Conservation

\section{INTRODUCTION}

MicroRNAs (miRNAs) are endogenous, non-coding small RNAs that regulate the flow of genetic information by controlling the translation or stability of mRNAs (Carrington and Ambros, 2003). It has been estimated that miRNAs account for $\sim 1 \%$ of predicted genes in higher eukaryotic genomes, and that up to $10-30 \%$ of genes may be regulated by miRNAs (Cui et al., 2006).

Since the discovery of the first miRNA, lin-4, in Caenorhabditis elegans (Lee et al., 1993), thousands of miRNAs have been identified by experimental cloning or computational approaches. Some miRNAs are difficult to find using cloning or deep sequencing, owing to their physical properties, including sequence composition, methylation, and post-transcriptional modifications (Berezikov et al., 2006). Therefore, computational approaches were widely used as a rapid and affordable method to overcome the limitation of experimental strategy (Zhang et al., 2006a). Many miRNAs are evolutionarily conserved within the same kingdom. Relying on its conservation, Zhang et al. (2005) developed a homology search-based approach for identifying plant miRNAs. Using this approach, more than 700 miRNAs have been identified in plants and viruses (Zhang et al., 2006a; Pan et al., 2007). Since there are a number of non-conserved miRNAs (Lu et al., 2005; Devor et al., 2009), the use of only the conserved miRNA sequences as the query set in the above approach would be more sensitive and specific for the detection of miRNA homologues. Furthermore, analysis of these conserved miRNAs and corresponding families may provide information concerning the phylogenetic distribution, species-specific miRNA family preference and conservation.

With the identification of increasing numbers of miRNAs and their targets, our current knowledge of their regulatory roles has spread over a large spectrum of plant developmental programs, including growth and developmental patterning, metabolic processes, hormone responses, stress defense, and signaling (Jones-Rhoades et al., 2006; Sunkar et al., 2007). Plant miRNAs were found to regulate gene expression by binding to targeted mRNAs in a perfect or near-perfect complementary site (Schwab et al., 2005; Axtell et al., 2007). This suggested that the miRNA-target modules should be conserved in long evolutionary timescales. As expected, earlier studies demonstrated that the target mRNAs of conserved miRNAs have a narrower range of functions than the targets of non-conserved miRNAs (Willmann and Poethig, 2007). Therefore, a comparative analysis of the target genes for ancient miRNA families may broaden our understanding of the conserved regulatory interactions within the plant kingdom.

To date, a total of 2043 miRNAs have been identified in the plant kingdom, and the information has been deposited in the miRBase database (Release 14: September 2009). In this study, we examined 42 conserved miRNA families from the miRBase database in an attempt to increase our knowledge of the phylogenetic distribution and preferential expression of miRNA genes. Furthermore, using an improved computational approach and 
the sequences of conserved miRNAs as the query set, 34 conserved miRNAs and 46 target genes were detected in wheat. Most conserved miRNAs have retained homologous target interactions except miR172.

\section{MATERIAL AND METHODS}

\section{Sequences of miRNAs, expressed sequence tag, genomic survey sequences, and mRNA}

All sequences of mature miRNAs and their precursors (pre-miRNAs) were downloaded from the miRBase database (Release 10.1, http://microrna.sanger.ac.uk/ sequences/index.shtml). This set contained 1467 miRNAs from 17 plant species: Arabidopsis thaliana, 184; Oryza sativa, 243; Physcomitrella patens, 220; Pinus taeda, 27; Populus trichocarpa, 215; Selaginella moellendorffii, 58; Sorghum bicolor, 72; Vitis vinifera, 100; Zea mays, 96; etc. On the basis of the conservation of miRNAs, 42 miRNA families present in more than one species were examined. The wheat expressed sequence tag (EST), genomic survey sequence (GSS), and mRNA databases were obtained from the NCBI database site (http://www.ncbi.nlm.nih.gov/).

\section{Prediction of conserved miRNAs in wheat}

Minimizing false positives is important for the identification of new miRNAs. The procedure used to search for conserved miRNAs in this study was essentially as described in previous studies (Nasaruddin et al., 2007; Zhang et al., 2007; Yin et al., 2008), but with some modifications (Supplementary Figure S1, A). Herein, to improve the sensitivity and specificity of the prediction, non-conserved miRNA sequences were eliminated. Only the conserved sequences were used as the query to perform BLAST searches against wheat EST and GSS databases. The EST or GSS sequences that have only 0-2 nucleotide (nt) mismatches compared with the query sequences were selected manually, and used to search against a protein database to remove the degradation products of protein-coding sequences. The remaining sequences were used to predict the hairpin structures by Mfold 3.2 (http://frontend.bioinfo.rpi. edu/applications/mfold/cgi-bin/rna-form1.cgi). Ultimately, redundant nucleotides were discarded to obtain the mature sequences of new miRNAs in wheat.

\section{Prediction of target genes and the analysis of their functional similarity}

The major steps and parameter settings for predicting target genes of miRNAs were performed as described in previous studies (Schwab et al., 2005; Yao et al., 2007; Supplementary Figure S1, B). Briefly, the miRNA sequences identified were used in a BLAST search against the wheat mRNA database. Sequences with only 0-4 nt mismatches compared with the query miRNA sequences were selected manually. The Unigene database contains many transcript sequences that appear to come from the same transcription locus, and information on protein similarities. There are 41,256 entries for wheat in the Unigene database, and closely related wheat mRNAs have been assembled in the Unigene cluster. Thus, data from the Unigene accessions and Arabidopsis Small RNA Project (ASRP, http://asrp.cgrb.oregonstate. $\mathrm{edu} / \mathrm{db} /$ ) were used to analyze the functional similarity of target genes. 


\section{RESULTS AND DISCUSSION}

\section{The conservation of plant miRNAs}

It is recognized that many miRNAs are evolutionarily conserved across species boundaries from mosses to eudicots. In this study, 42 conserved miRNA families that are present in more than one plant species were examined from the miRBase database (Table 1). Among them, 8 families (miR156/157, miR159, miR160, miR166, miR167, miR171, miR319, and miR396) are present in more than 10 species; 12 families are present in 6 to 10 species, and the remaining 22 families are present in 2 to 5 species.

Table 1. The number of land plant species that contain a conserved microRNA (miRNA) family and the
corresponding conserved miRNA families.
\begin{tabular}{ll}
\hline Number of plant species & Conserved miRNA family \\
\hline $\begin{array}{ll}11-17 \\
6-10\end{array}$ & $\begin{array}{l}\text { miR156/157 miR159 miR160 miR166 miR } 167 \text { miR171 miR319 miR396 } \\
\text { miR162 miR164 miR168 miR169 miR172 miR390 miR393 miR394 miR395 miR398 miR399 miR408 } \\
\text { miR161 miR397 miR403 miR413 miR414 miR415 miR416 miR417 miR418 miR419 miR420 miR426 } \\
\text { miR444 miR472 miR477 miR479 miR482 miR529 miR535 miR536 miR783 miR824 }\end{array}$
\end{tabular}

All families are present in more than one plant species in the miRBase database (Release 10.1).

During screening of conserved miRNA families, we found that the conserved miRNAs mainly exist in dicots and monocots, and are less frequent in ferns and mosses. The proportion of conserved miRNAs is 0.823 and 0.614 in poplar and rice, respectively. However, the proportion in S. moellendorffii and P. patens is only 0.281 and 0.264 , respectively. Since the bryophytes are the most ancient land plants, this observation seems in contrast to the hypothesis that the conservation of miRNAs is the result of shared ancestry or functional convergence from an independent origin during evolution (Maher et al., 2006). One possible explanation is that miRNA genes were subjected to strong purifying selection during evolution, and that some were lost, while others experienced multiple rounds of duplication (Jiang et al., 2006; Fahlgren et al., 2007; Axtell, 2008).

To analyze the preferential expression of miRNAs, we plotted the number of miRNA members in each conserved family that are found in the sequenced (or almost fully sequenced) genomes for dicots and monocots. We used Arabidopsis, poplar and grape for the dicots, and rice, sorghum and maize for the monocots. We found that most miRNA families have an equal number of members between dicots and monocots. In a few species, miRNA families were preferentially expressed in dicots relative to monocots, especially miR403, miR472 and miR479 (Figure 1). This specific distribution may be indicative of their specific function in miRNA-mediated gene regulation in dicots, although the details of this process are unknown.

\section{Identification of conserved miRNAs in wheat}

In order to improve the sensitivity and specificity of miRNA prediction, we eliminated non-conserved plant miRNA sequences, and used only the sequences of 42 conserved miRNA families described above as the query set. Following a set of strict filtering criteria, a total of 34 conserved miRNAs were detected in wheat. They were classified into 18 families on the 


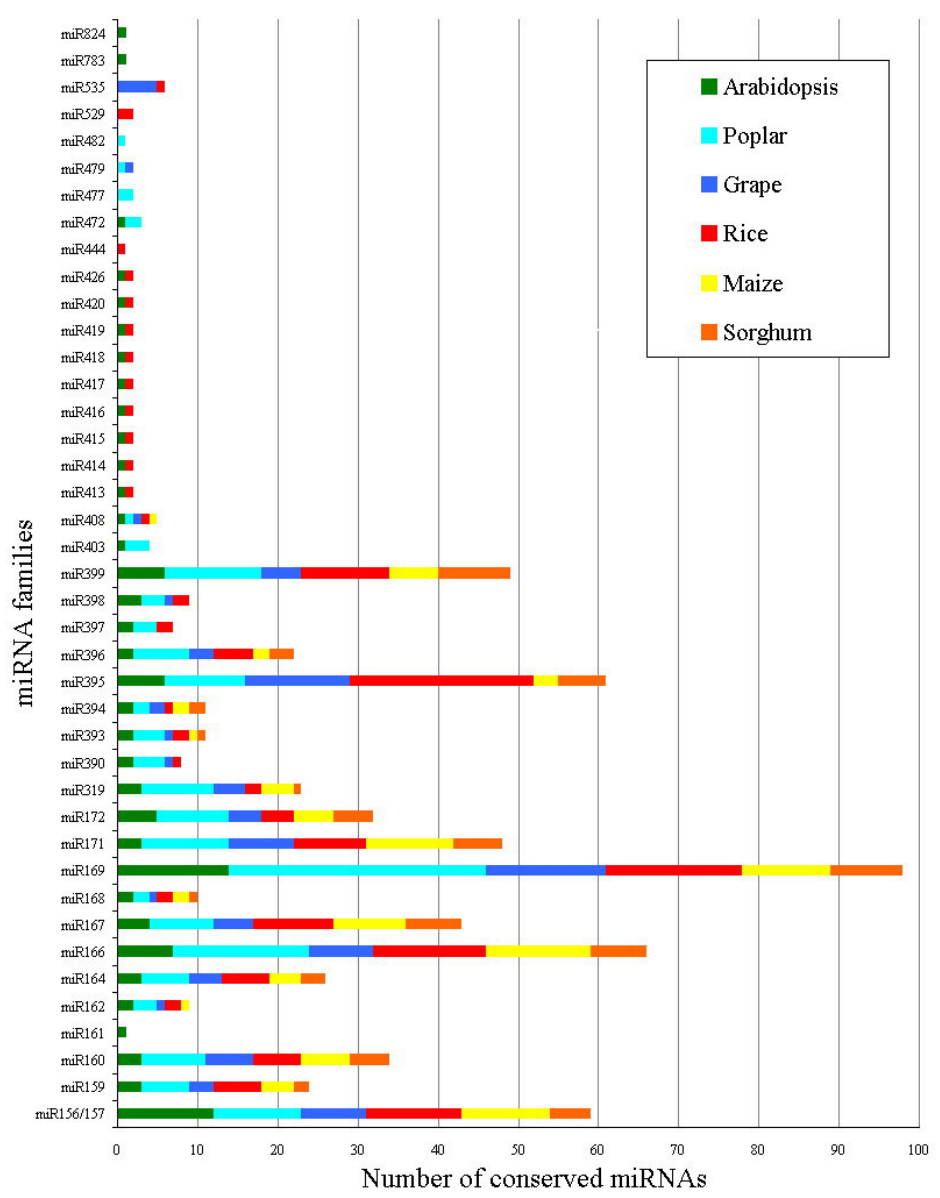

Figure 1. The number of miRNAs in each conserved family (dicots, green/greenish/blue; monocots, red/ yellow/reddish).

basis of sequence similarity (Table 2). The mature sequences of 25 miRNAs begin with 5 '-uridine, a characteristic feature of plant miRNAs. Among the 34 wheat miRNAs identified, 14 have the same nucleotide sequences as the query miRNAs. This suggests that these miRNAs are more highly conserved than others.

Considering that computational approaches or high-throughput sequencing have identified many wheat miRNAs, the mature sequences of the 34 identified miRNAs were compared with published data for miRNAs (Yao et al., 2007; Dryanova et al., 2008; Jin et al., 2008; Han et al., 2009; Wei et al., 2009). BLAST results show that 27 miRNAs have identical sequence with previously reported miRNAs in wheat. The miR482 family and the other miRNAs, including miR172b, miR319b, miR414c, miR419, and miR444b, were newly identified. The precursors of newly identified miRNAs had a high minimal folding free energy 
Table 2. MicroRNAs (miRNAs) in wheat.

\begin{tabular}{|c|c|c|c|c|c|}
\hline miRNA & ID & Sequence & End/Source & $\mathrm{A}+\mathrm{U}(\%)$ & MFEI \\
\hline miR156a & 51666269 & ugacagaagagagugagcac & $5^{\prime} / \mathrm{GSS}$ & 46.43 & 0.925 \\
\hline $\operatorname{miR} 156 b$ & 20120267 & cugacagaagaGagagagcaU & $5^{\prime} / \mathrm{EST}$ & 50.12 & 0.712 \\
\hline $\operatorname{miR} 156 \mathrm{c}$ & 20122061 & ugacagaagaGagagagcac & $5^{\prime} / \mathrm{EST}$ & 50.12 & 0.754 \\
\hline $\operatorname{miR} 159$ & 93066835 & uuggaAugaagggagcucca & $3 \prime$ EST & 45.82 & 0.744 \\
\hline miR160a & 93162853 & ugccuggcucccuguaugeca & $5^{\prime} / \mathrm{EST}$ & 36.67 & 0.909 \\
\hline $\operatorname{miR} 160 \mathrm{~b}$ & 93237563 & ugccuggcucccuguaugec $\mathrm{U}$ & $5^{\prime} / \mathrm{EST}$ & 39.69 & 0.738 \\
\hline $\operatorname{miR} 164$ & 25426214 & uggagaagcagggcacgugca & $5^{\prime} / \mathrm{EST}$ & 30.26 & 0.898 \\
\hline $\operatorname{miR} 167 a$ & 143399055 & ugaagcugccagcaugaucua & $5^{\prime} / \mathrm{EST}$ & 48.68 & 0.921 \\
\hline $\operatorname{miR} 169 a$ & 19956307 & uagccCaggGugacuugccca & $5^{\prime} / \mathrm{EST}$ & 39.39 & 0.889 \\
\hline $\operatorname{miR} 169 b$ & 93186556 & aagccaaggaugaGuugccug & $5^{\prime} / \mathrm{EST}$ & 40.37 & 0.701 \\
\hline $\operatorname{miR} 171$ & 32685227 & ugauugagecgugccaauauc & 3'/EST & 38.30 & 0.816 \\
\hline $\operatorname{miR} 172 \mathrm{a}$ & 9696528 & aAaauccugaugaugcugcaG & $5^{\prime} / \mathrm{EST}$ & 40.75 & 0.726 \\
\hline miR172b & 143362423 & AgGaucuugaugaugcugcag & $5^{\prime} / \mathbf{E S T}$ & 61.11 & 0.781 \\
\hline $\operatorname{miR} 319 a$ & 24978824 & uuuggaUugaagggagcucU & $3 ' / \mathrm{EST}$ & 52.80 & 0.927 \\
\hline miR319b & 24977949 & GuggacugaagUgagcucceu & $3^{\prime} / \mathbf{E S T}$ & 48.73 & 0.922 \\
\hline $\operatorname{miR} 319 c$ & 25440767 & cuuggacugaagUgagcuAc & 3'/EST & 32.33 & 0.740 \\
\hline miR319d & 75684012 & uuggacugaagggagcucccu & $3^{\prime} / \mathrm{GSS}$ & 48.99 & 0.965 \\
\hline $\operatorname{miR} 395 a$ & 39556435 & augaaguguuugggggaacuc & $3^{\prime} / \mathrm{EST}$ & 56.75 & 0.909 \\
\hline $\operatorname{miR} 395 b$ & 55605069 & ugaaguguuugggggaacuc & $3^{\prime} / \mathrm{EST}$ & 54.55 & 1.162 \\
\hline $\operatorname{miR} 395 \mathrm{c}$ & 39556094 & uugaaguguuugggggaacuc & $5^{\prime} / \mathrm{EST}$ & 57.41 & 0.942 \\
\hline $\operatorname{miR} 396$ & 141574195 & uccacaggcuuucuugaacug & $5^{\prime} / \mathrm{EST}$ & 45.26 & 0.808 \\
\hline $\operatorname{miR} 398$ & 93224516 & uguguucucaggucgececCgC & $3^{\prime} / \mathrm{EST}$ & 44.19 & 0.923 \\
\hline $\operatorname{miR} 399 a$ & 93255560 & ugccaaaggagaguugccc & $3^{\prime} / \mathrm{EST}$ & 41.33 & 0.925 \\
\hline $\operatorname{miR} 399 b$ & 93234499 & ugccaaaggagaauugccc & $3^{\prime} / \mathrm{EST}$ & 40.35 & 0.899 \\
\hline $\operatorname{miR} 399 c$ & 93057089 & ugccaaaggagaguugcccug & $3^{\prime} / \mathrm{EST}$ & 41.77 & 0.976 \\
\hline $\operatorname{miR} 414 a$ & 93058127 & ucaucAucaucaucaucgucG & 3'/EST & 41.01 & 0.727 \\
\hline $\operatorname{miR} 414 b$ & 93270953 & ucaucAucaucaucGucguca & $5^{\prime} / \mathrm{EST}$ & 40.42 & 0.741 \\
\hline $\operatorname{miR414c}$ & 93236205 & ucauccucaucCucaucgucc & $3^{\prime} / \mathbf{E S T}$ & 47.62 & 0.719 \\
\hline $\operatorname{miR} 415$ & 92237256 & GCcagagcagaaacagaacau & $3^{\prime} / \mathrm{EST}$ & 52.17 & 0.832 \\
\hline $\operatorname{miR419}$ & 39002573 & AuaugaaugcugaggUuguug & $5^{\prime} / \mathbf{E S T}$ & 61.54 & 0.975 \\
\hline $\operatorname{miR} 444 a$ & 39562974 & uugcugccucaagcuugcuge & $3^{\prime} / \mathrm{EST}$ & 50.33 & 1.021 \\
\hline miR444b & 70966362 & uugcugUcucaagcuugcugA & 3'/EST & 46.02 & 0.919 \\
\hline miR482a & 29168987 & ucuucceAacuccСeccauucc & $\mathbf{3}^{\prime} / \mathbf{E S T}$ & 52.60 & 0.727 \\
\hline miR482b & 22099570 & ucuucecСacuccСeccauucc & $3^{\prime} / \mathbf{E S T}$ & 45.24 & 0.722 \\
\hline
\end{tabular}

The miRNAs newly identified in wheat are shown in bold. The capital letters in sequences represent the mismatched nucleotides. MFEI = minimal folding free energy index; GSS = genomic survey sequence; EST $=$ expressed sequence tag.

index of 0.719-0.975 (Table 2), which is significantly higher than those reported for tRNAs (0.64), rRNAs (0.59), and mRNAs (0.62-0.66) (Zhang et al., 2006b). The diverse fold-back of pre-miRNAs required a length of 41-462 nt (Supplementary Figure S2), which is similar to findings in other plant species.

\section{Conserved miRNA-target interactions in wheat}

Gaining insight into miRNA target genes can shed light on the range of miRNA regulation and can lead to a detailed description of miRNA-target interactions. Herein, a total of 46 target genes were detected in the wheat mRNA database (Table 3). Subsequently, the entries in the Unigene database and ASRP were selected to examine the functional similarity of predicted target mRNAs.

Most target mRNAs in wheat were similar or related to previously validated miRNA targets in Arabidopsis, rice, and popular. For instance, squamosa-promoter binding proteinlike (SPL) genes were predicted to be targeted by miR156. This consists of the recent demonstration that miR156 target members of the SBP-box gene family in both mono- and di- 
Table 3. The target genes of wheat microRNAs (miRNAs).

\begin{tabular}{|c|c|c|c|}
\hline miRNA & Targeted protein & Target genes & $\begin{array}{c}\text { Conserved with } \\
\text { Arabidopsis or } \\
\text { other plant species }\end{array}$ \\
\hline \multirow[t]{4}{*}{ tae-miR156 } & SPL2 & Та.3711 & Yes \\
\hline & SPL3 & Та.6374 & Yes \\
\hline & SPL11 & Та.7021 Та.67187 & Yes \\
\hline & Unknown & AL810223 CJ653710 & \\
\hline \multirow[t]{2}{*}{ tae-miR $159 / 319$} & MYB domain protein & Та.24098 Та.49649 Та.58485 & Yes \\
\hline & Unknown protein & Ta.2427 Ta.58485 Ta.29557 DR737225 & \\
\hline \multirow[t]{2}{*}{ tae-miR160 } & Auxin response factor 10 & Ta. 13246 & Yes \\
\hline & Auxin response factor 16 & Та.35774 Та.44707 & Yes \\
\hline tae-miR164 & NAC domain containing protein & Тa.33080 & Yes \\
\hline \multirow{2}{*}{ tae-miR167 } & Auxin response factor 6 & Ta.9550 Тa.38988 Та.63221 & Yes \\
\hline & Auxin response factor 8 & Ta.6394 & Yes \\
\hline \multirow[t]{2}{*}{ tae-miR169 } & CBF-B/NF-YA family protein & Ta.10047 Тa.48680 Тa.57252 & Yes \\
\hline & & Ta.49366 Ta.38584 & \\
\hline \multirow[t]{2}{*}{ tae-miR171 } & Scarecrow-like transcription factor (SCL) & Ta.39354 & Yes \\
\hline & Hypothetical protein & Тa.36668 & \\
\hline \multirow[t]{3}{*}{ tae-miR172 } & TARGET OF EAT (Apetala2-like protein) & Тa.13336 Та.24445 & Yes \\
\hline & Succinyl-CoA ligase (GDP-forming) beta-chain & Ta.14585 & No \\
\hline & Unknown protein & Ta.11232 & \\
\hline \multirow[t]{2}{*}{ tae-miR395 } & ATP sulfurylase & Ta.9352 & Yes \\
\hline & Unknown protein & Ta.39519 CV779320 & \\
\hline tae-miR396 & Growth-regulating factor 3 & Ta.36890 & Yes \\
\hline tae-miR399 & Unknown protein & Ta.43848 CK210056 & \\
\hline tae-miR414 & Unknown protein & Тa.23809 Та.38775 Та.6362 & \\
\hline tae-miR415 & $\begin{array}{l}\text { Aminoacylase, putative/N-acyl-L-amino-acid } \\
\text { amidohydrolase }\end{array}$ & Ta.9380 & \\
\hline \multirow[t]{2}{*}{ tae-miR482 } & Quinolinate synthetase & Та.35819 & \\
\hline & Unknown protein & Ta.26024 Тa.13326 Тa.24321 & \\
\hline
\end{tabular}

cots, and have a conserved role as positive regulators of the vegetative to reproductive phase transition (Schwarz et al., 2008). Auxin response factors were predicted as targets of miR160 and miR167. In addition, other miRNA-target interactions were also conserved in wheat as expected, such as miR171 - SCL (scarecrow-like transcription factor), miR395 - ATP sulfurylase, miR396 - growth-regulating factor, etc. (Table 3). Among the targets, the additional targeting of succinyl-CoA ligase by miR172 was of particular interest. In the plant kingdom, miR172 have been well known to TARGET OF EAT (TOE) transcription factors (Mlotshwa et al., 2006). Herein, the mRNAs of succinyl-CoA ligase have a complementary site similar to that of TOE, as illustrated in Figure 2. This novel phenomenon suggested that species-specific regulations for miR172 may exist in wheat. In popular, Lu et al. (2005) found that ptr-miR156 target not only SPL genes but also nitrate transporter, exhibiting a popular-specific function. These observations suggest that the function of some well-conserved miRNAs have drifted during long periods of plant evolution. The recognition of target genes by miRNAs requires a high degree of base-pairing between miRNA sequence and target sites. Although miRNAs are well conserved in long evolutionary timescales, some of their sequences have changed and display variations in a few nucleotide positions (Axtell and Bowman, 2008). It provides the chance for some miRNAs to base pair with other target mRNAs, exhibiting species-specific regulatory pattern.

In summary, 42 conserved miRNA families were examined from the miRBase database. The distribution analysis showed that some conserved miRNA families have a preferential expression in dicots relative to monocots, especially miR403, miR472 and miR479. Subsequently, 34 conserved miRNAs were identified in wheat. Five miRNAs (miR172b, 


\author{
TOE Ta.13336 \\ 5'-UCAUCAUCCGCUGCAGC AUCAUCAGGAUUCUCCAAAACCGC-3' \\ ||||||||||||||०||| O | \\ 3'-GACGUCGUAGUAGUUCUAGGA-5' 172b \\ Succinyl-CoA ligase Ta.14585 \\ 5'-ACAGCCUUCUCUGCAGCA UCAUCAAGAUCCUCUGCAGUGAU-3' \\ |||||||||||||||||| $\mid$ \\ 3'-GACGUCGUAGUAGUUCUAGGA-5' 172b
}

Figure 2. Two predicted target mRNAs of miR172. Succinyl-CoA ligase mRNAs has a complementary site similar to that of TOE mRNAs, which are targeted by miR172.

miR319b, miR414c, miR419, and miR444b) and the miR482 family were newly identified. Most of the conserved wheat miRNAs have retained homologous target interactions throughout plant evolution. However, miR172 display a wheat-specific function and have an additional target interaction with succinyl-CoA ligase. These results suggest that the expression and function of some conserved miRNAs have drifted during long evolutionary timescales. The next major step will be to experimentally verify the above observations. The identification of these miRNAs and target genes reveal the future path leading to the understanding of the core regulatory interactions in wheat.

\title{
ACKNOWLEDGMENTS
}

Research supported by China's Key Development Project for Basic Research (973) (Grant \#2007CB116208), and China's Major Projects for Transgenic Breeding (Grants \#2008ZX005-004 and \#2008ZX08005-002).

\section{REFERENCES}

Axtell MJ (2008). Evolution of microRNAs and their targets: are all microRNAs biologically relevant? Biochim. Biophys. Acta 1779: 725-734.

Axtell MJ and Bowman JL (2008). Evolution of plant microRNAs and their targets. Trends Plant Sci. 13: 343-349.

Axtell MJ, Snyder JA and Bartel DP (2007). Common functions for diverse small RNAs of land plants. Plant Cell 19: 1750-1769.

Berezikov E, Cuppen E and Plasterk RH (2006). Approaches to microRNA discovery. Nat. Genet. 38 (Suppl): S2-S7.

Carrington JC and Ambros V (2003). Role of microRNAs in plant and animal development. Science 301: 336-338.

Cui Q, Yu Z, Purisima EO and Wang E (2006). Principles of microRNA regulation of a human cellular signaling network. Mol. Syst. Biol. 2: 46.

Devor EJ, Peek AS, Lanier W and Samollow PB (2009). Marsupial-specific microRNAs evolved from marsupial-specific transposable elements. Gene 448: 187-191.

Dryanova A, Zakharov A and Gulick PJ (2008). Data mining for miRNAs and their targets in the Triticeae. Genome 51: 433-443.

Fahlgren N, Howell MD, Kasschau KD, Chapman EJ, et al. (2007). High-throughput sequencing of Arabidopsis microRNAs: evidence for frequent birth and death of MIRNA genes. PLoS. One 2: e219.

Han Y, Luan F, Zhu H, Shao Y, et al. (2009). Computational identification of microRNAs and their targets in wheat (Triticum aestivum L.). Sci. China C. Life Sci 52: 1091-1100. 
Jiang D, Yin C, Yu A, Zhou X, et al. (2006). Duplication and expression analysis of multicopy miRNA gene family members in Arabidopsis and rice. Cell Res. 16: 507-518.

Jin W, Li N, Zhang B, Wu F, et al. (2008). Identification and verification of microRNA in wheat (Triticum aestivum). J. Plant Res. 121: 351-355.

Jones-Rhoades MW, Bartel DP and Bartel B (2006). MicroRNAS and their regulatory roles in plants. Annu. Rev. Plant Biol. 57: 19-53.

Lee RC, Feinbaum RL and Ambros V (1993). The C. elegans heterochronic gene lin-4 encodes small RNAs with antisense complementarity to lin-14. Cell 75: 843-854.

Lu S, Sun YH, Shi R, Clark C, et al. (2005). Novel and mechanical stress-responsive microRNAs in Populus trichocarpa that are absent from Arabidopsis. Plant Cell 17: 2186-2203.

Maher C, Stein L and Ware D (2006). Evolution of Arabidopsis microRNA families through duplication events. Genome Res. 16: 510-519.

Mlotshwa S, Yang Z, Kim Y and Chen X (2006). Floral patterning defects induced by Arabidopsis APETALA2 and microRNA172 expression in Nicotiana benthamiana. Plant Mol. Biol. 61: 781-793.

Nasaruddin NM, Harikrishna K, Othman RY, Lim SH, et al. (2007). Computational prediction of microRNAs from Oil Palm (Elaeis guineensis Jacq.) expressed sequence tags. Asia Pac. J. Mol. Biol. Biotechnol. 15: 107-113.

Pan X, Zhang B, San Francisco M and Cobb GP (2007). Characterizing viral microRNAs and its application on identifying new microRNAs in viruses. J. Cell Physiol. 211: 10-18.

Schwab R, Palatnik JF, Riester M, Schommer C, et al. (2005). Specific effects of microRNAs on the plant transcriptome. Dev. Cell 8: 517-527.

Schwarz S, Grande AV, Bujdoso N, Saedler H, et al. (2008). The microRNA regulated SBP-box genes SPL9 and SPL15 control shoot maturation in Arabidopsis. Plant Mol. Biol. 67: 183-195.

Sunkar R, Chinnusamy V, Zhu J and Zhu JK (2007). Small RNAs as big players in plant abiotic stress responses and nutrient deprivation. Trends Plant Sci. 12: 301-309.

Wei B, Cai T, Zhang R, Li A, et al. (2009). Novel microRNAs uncovered by deep sequencing of small RNA transcriptomes in bread wheat (Triticum aestivum L.) and Brachypodium distachyon (L.) Beauv. Funct. Integr. Genomics 9: 499511.

Willmann MR and Poethig RS (2007). Conservation and evolution of miRNA regulatory programs in plant development. Curr. Opin. Plant Biol. 10: 503-511.

Yao Y, Guo G, Ni Z, Sunkar R, et al. (2007). Cloning and characterization of microRNAs from wheat (Triticum aestivum L.). Genome Biol. 8: R96.

Yin Z, Li C, Han X and Shen F (2008). Identification of conserved microRNAs and their target genes in tomato (Lycopersicon esculentum). Gene 414: 60-66.

Zhang BH, Pan XP, Wang QL, Cobb GP, et al. (2005). Identification and characterization of new plant microRNAs using EST analysis. Cell Res. 15: 336-360.

Zhang B, Pan X, Wang Q, Cobb GP, et al. (2006a). Computational identification of microRNAs and their targets. Comput. Biol. Chem. 30: 395-407.

Zhang BH, Pan XP, Cox SB, Cobb GP, et al. (2006b). Evidence that miRNAs are different from other RNAs. Cell Mol. Life Sci. 63: 246-254.

Zhang B, Wang Q, Wang K, Pan X, et al. (2007). Identification of cotton microRNAs and their targets. Gene 397: $26-37$. 


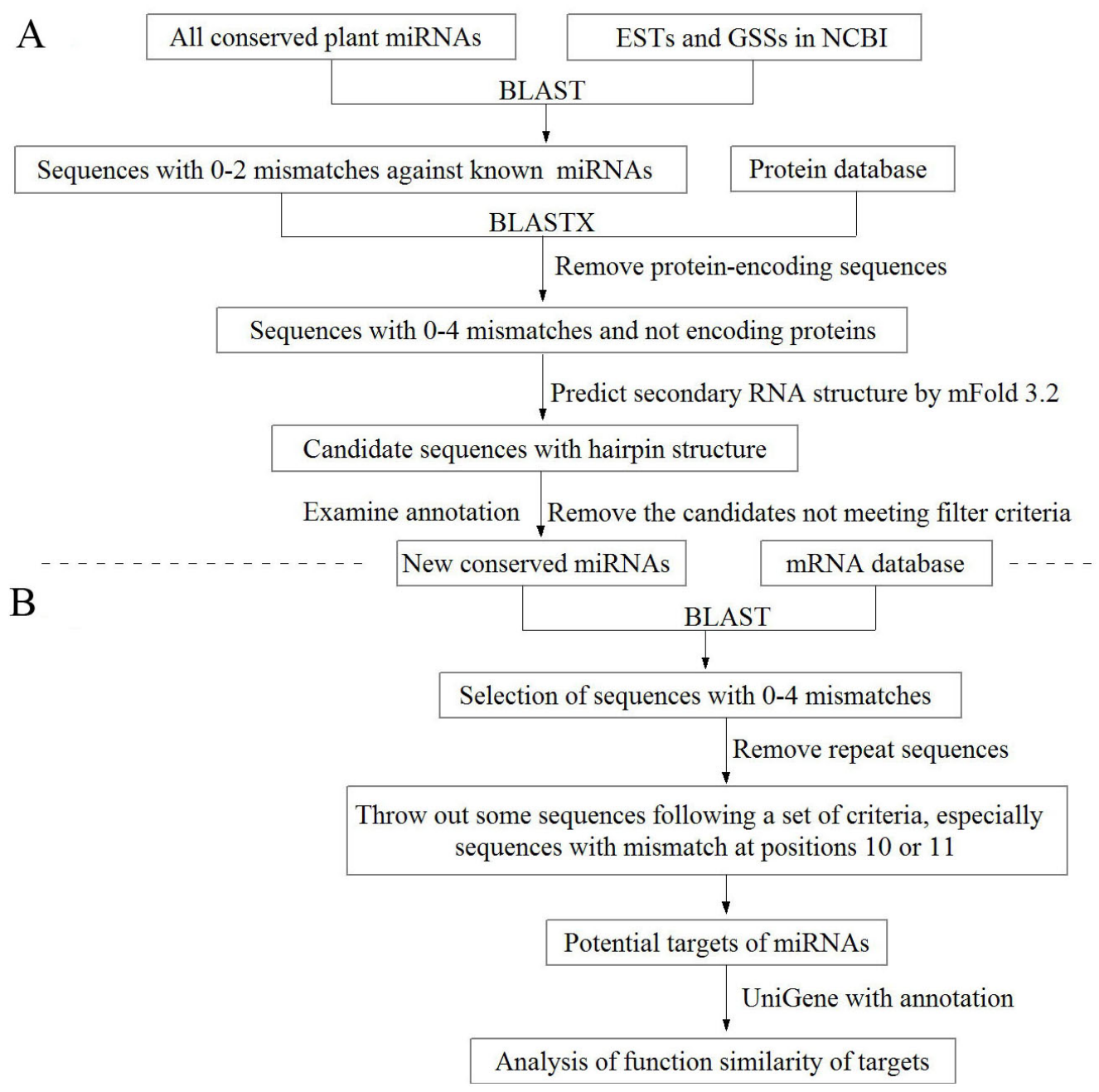

Supplementary Figure S1. Flowchart for the prediction of conserved miRNAs and their target genes in wheat. A. Schematic representation for the prediction of conserved miRNAs. B. Schematic representation for the prediction of target genes of the miRNAs identified. 


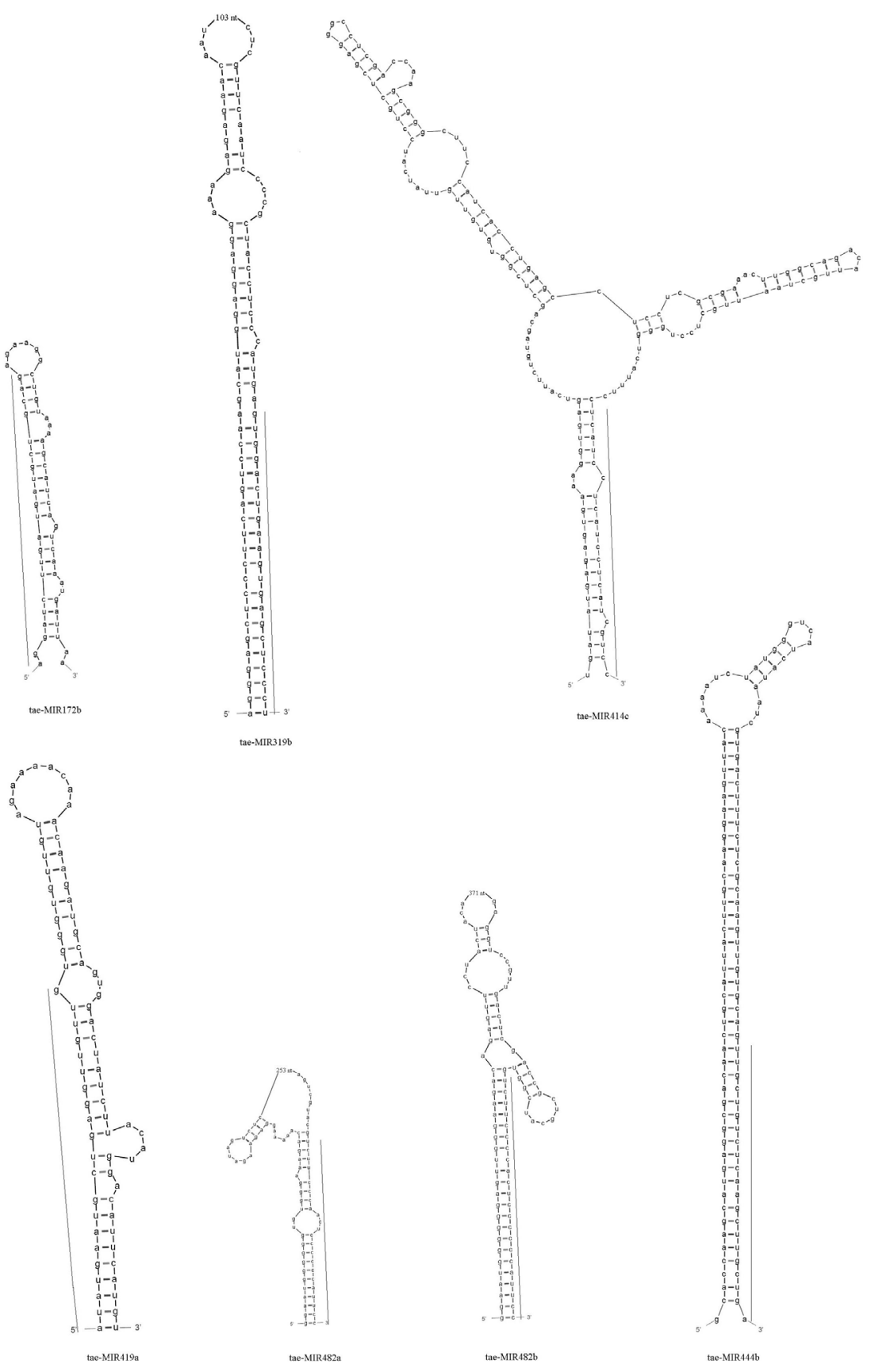

Supplementary Figure S2. Predicted fold-back structures of the newly identified 7 miRNA precursors in wheat. Sequences of mature miRNAs are underlined. 